\title{
The effect of transcutaneous vagus nerve stimulation on fear generalization and subsequent fear extinction
}

Burger, A.M. ${ }^{a, b} *$

Van Diest, I. ${ }^{b}$

Van der Does, W. ${ }^{a}$

Korbee, J.N. ${ }^{a}$

Waziri, N. ${ }^{a}$

Brosschot, J.F. ${ }^{\text {a }}$

Verkuil, B. ${ }^{a}$

${ }^{a}$ Institute of Psychology, Leiden University; Wassenaarseweg 52, 2333 AK Leiden, The Netherlands.

${ }^{b}$ Faculty of Psychology, Katholieke Universiteit Leuven; Tiensestraat 102, 3000 Leuven, Belgium.

*Andreas M. Burger is the Corresponding Author for this manuscript. His email address is a.m.burger@fsw.leidenuniv.nl. 


\begin{abstract}
Fear overgeneralization is thought to be one of the cardinal processes underlying anxiety disorders, and a determinant of the onset, maintenance and recurrence of these disorders. Animal studies have shown that stimulating the vagus nerve (VNS) affects neuronal pathways implicated in pattern separation and completion, suggesting it may reduce the generalization of a fear memory to novel situations. In a one-day study, 58 healthy students were subjected to a fear conditioning, fear generalization, and fear extinction paradigm. Participants were randomly assigned to receive either transcutaneous auricular VNS (tVNS; final $N=29$ ) or sham stimulation (final $N=29$ ) during the generalization and extinction phases. tVNS did not affect fear generalization, as reflected by US expectancy ratings and fear potentiated startle responses. However, participants who received tVNS reported lower US expectancy ratings to the CS+ during the extinction phase, possibly reflecting a stronger declarative extinction of fear. No effects of tVNS on fear potentiated startle responses during extinction were found. The pattern of findings regarding extinction of declarative fear suggest a facilitating effect of $t V N S$.
\end{abstract}




\section{Introduction}

Associative learning is fundamental for survival, as it enables us to understand the relationship between stimuli, contexts, or actions, and outcomes. A key aspect of associative learning is the ability to generalize what has been learned to new situations, allowing individuals to discern threatening from non-threatening stimuli or contexts through unconscious inductive processes. The downside of generalized learning appears when individuals overgeneralize fear towards cues or contexts that are typically safe. Indeed, the over-generalization of threat towards cues and contexts that are neutral or actually safe has been proposed to be strongly associated with the onset as well as the maintenance of anxiety disorders [1-3].

Memory generalization can be conceptualized as a result of interference resolution between novel stimuli or contexts and similar memories. The resolution of this interference is critically dependent on subregions within the hippocampus, where the neuronal pattern of the novel memory trace is compared to the pattern of other memory traces (for reviews on this subject, see $[4,5])$. In case of a large overlap between these neuronal representations, CA3 neurons in the hippocampus are thought to initiate a process of 'pattern completion', where the representational overlap between the memory patterns is increased, leading to generalization of the previous memory trace [6]. Alternatively, in case of a small overlap between neuronal representations, neurons in the dentate gyrus (DG) and the CA3 of the hippocampus initiate a process of 'pattern separation', inhibiting subsequent memory generalization [6]. The importance of the DG for the inhibition of fear generalization has repeatedly been demonstrated in animal studies. Rats with inactive DG receptors either due to hippocampal lesions or genetic abolition exhibited normal levels of freezing during fear conditioning, but were less able to inhibit freezing responses in similar contexts, indicating that they were unable to separate their neuronal representations of the novel and the previously learned threatening context [7-11]. In line with these findings, high-resolution fMRI in humans showed increased BOLD activity in the CA3/DG during pattern seperation of highly similar cues [12]. Additionally, during a cue fear conditioning study in humans, participants demonstrated stronger BOLD activity in the hippocampus as an inverse function of perceptual similarity between generalized stimuli and the feared stimulus, possibly 
reflecting stronger pattern separation for stimuli that are more easily discernable from the feared stimulus [13]. Conversely, stronger functional connectivity of the hippocampus with the amygdala and the insula - brain areas that are involved in fear excitation - was found for stimuli that resembled the CS+, confirming a role of the hippocampus in pattern completion [13]. In sum, the hippocampus, and specifically the DG, seems to be an important determinant of fear generalization in both animal and human literature. Interventions aimed at increasing activity in the DG may affect individuals' ability to discriminate between novel and feared cues or contexts, and could therefore constitute a promising add-on to psychological treatment.

Vagus nerve stimulation (VNS) has recently drawn attention as a potential add-on for exposure therapy. VNS is thought to affect learning and memory by activating the locus coeruleus to secrete norepinephrine (NE) to fear- and learning-related brain areas, including the hippocampus [14]. Studies in rats demonstrated that cutting the afferent fibers of the vagus attenuated cued but not contextual extinction learning [15], whereas invasively stimulating the vagus nerve strengthened the extinction of auditory conditioned fear [1619]. In humans, transcutaneous, non-invasive stimulation of the auricular branch of the vagus nerve (tVNS) has been found to accelerate declarative fear extinction in a cue conditioning paradigm [20,21], although this effect has not been found consistently [22] and has not been found in a context conditioning paradigm [23]. VNS may potentially also facilitate the inhibition of fear generalization, as secretion of NE is positively correlated to pattern separation in humans [24]. Additionally, animal studies showed that acute VNS increases cell proliferation in the DG of the hippocampus $[25,26]$, while both acute and chronic VNS increased dendrite complexity of neurons in the DG, indicating stronger neuronal plasticity [26]. Finally, one study found that VNS ameliorated the decreases in DG cell count that occurs after the bilateral removal of the olfactory bulbs, which is a validated animal model in depression research [27]. In sum, these studies indicate, at least in animal models, a clear effect of VNS on hippocampal DG activity and morphology, which may indicate that VNS may also strengthen the associated process of pattern seperation and thereby decrease fear generalization.

The effects of tVNS on the generalization of fear have been tested in one controlled study (supplementary in [20]), which found no significant differences between groups on the 
generalization of fear. However, that study had been primarily designed to assess the effects of tVNS on fear extinction, and the generalization of fear had been tested as a secondary outcome after extinction and retention had already taken place. Additionally, as the study was primarily designed to test the effects of tVNS on fear extinction, participants only received tVNS during the extinction phase on day 2 , and not during the generalization test 24h later.

In this study, we aim to test the hypothesis that tVNS decreases the generalization of fear. We hypothesize that participants who receive tVNS will show a steeper downwards generalization gradient than participants who receive sham stimulation. Additionally, we aim to perform a conceptual replication of previous studies $[20,21]$ on the effects of tVNS on fear extinction, where we hypothesize that tVNS accelerates the extinction of fear. To test these hypotheses, we conducted a randomized single-blinded controlled trial to compare the effects of tVNS and sham stimulation during the generalization and extinction of fear. Fear was measured on both a declarative level through the use of US expectancy ratings as well as on a physiological level through the use of fear potentiated startle responses. Startle responses are among the most commonly used physiological indices of fear, because of the critical involvement of the amygdala in the amplitude of the startle expression. Compared to skin conductance responses, they have the added benefit of having high temporal specificity, and return to baseline levels quickly after threat has subsided [28,29]. Participants were subjected to a fear conditioning paradigm based on Lissek and colleagues [13]. 


\section{Methods}

\subsection{Participants}

Sixty-two healthy college students between the ages 18 and 25 participated in the experiment. Exclusion criteria for the study were epilepsy, cardiac diseases, significant head trauma, pregnancy, drug use, or any neurological or psychiatric disorder.

Ethical approval for this study was given by the ethical committee of the Institute of Psychology of Leiden University (CEP\#16-1102/339). Participants were rewarded with either 10 euros or course credit.

\section{2. $\quad$ Procedure}

After participants provided informed consent, the electrodes for ECG and EMG measurements were attached. Next, the shock electrode was attached to the forearm of the non-dominant hand and the shock intensity was individually calibrated. Specifically, the shock was individually calibrated to an intensity that was 'highly uncomfortable, but not painful'. Participants received shocks of gradually increasing intensity, starting at $1 \mathrm{~mA}$ and increasing in 5mA increments. After every shock, participants were asked to rate whether the intensity could be increased, or whether it was already at a level that was "highly uncomfortable, but not painful'. After participants reported that the shock intensity had reached the desired level, the shock intensity was kept stable for the rest of the experiment.

Participants were told that during the computer tasks they would see different pictures, and it was their task to learn to predict whether they'd receive a shock based on what picture was being presented to them. As such, we gave participants partial instructions on CS-US contingencies. These kinds of partial instructions lead to more uniform fear learning curves compared to uninstructed procedures, while still leaving enough room for associative learning to take place [30].

Prior to the start of the acquisition phase, a five-minute baseline measurement of every participant's RMSSD level was recorded as a measure of vagally-mediated HRV. 
Afterwards, participants received instructions via the computer monitor about what stimuli would appear on screen during the rest of the experiment and how to rate shock expectancies. Participants were also presented with 10 startle probes over a period of 150 seconds to habituate startle blink responses. During this period, participants were also habituated to background noise (70dB pink noise), which would stay on for the remainder of the Acquisition, Generalization and Extinction phases (cf. [31]).

During the acquisition phase, both the CS+ and the CS- were presented 12 times. The CS+ was followed by the US in $75 \%$ of trials, the CS- was never followed by a shock. During the acquisition phase as well as all subsequent phases, trials were presented in a quasirandomized order, to ensure that one CS type could not be presented on more than 2 subsequent trials.

After the acquisition phase, we attached the tVNS device to the ear of the participant and we started either tVNS or sham stimulation. Participants were randomly assigned to receive either tVNS or sham stimulation. They were told that stimulation was expected to affect physiological processes during the tasks, and wore the nerve stimulator throughout the rest of the experimental procedure. With the tVNS device in place and active, participants completed a short demographics questionnaire and several questionnaires before starting the final task. This period, lasting roughly 10 minutes, was used as a 'ramp up' period for tVNS. Although not much is known about the temporal latency of potential learning effects of tVNS, a recent fMRI study has shown that effects of tVNS on hippompcal activity occur and plateau within roughly 6 minutes after stimulation onset [32]. As such, having a 10 minute period between stimulation onset and the subsequent generalization phase should leave enough time for tVNS to have an effect.

The generalization phase consisted of 12 presentations of the CS+ and CS-, and 6 presentations of every intermediate-sized GS. Thus, participants were presented with 12 CS+, 12 CS-, and 48 intermediate-sized GS circles during the generalization phase. During this phase, the CS+ was still followed by a shock in $50 \%$ of trials.

The generalization phase segued into the extinction phase, where the CS+ and CSwere presented another 10 times. No GS were presented during this phase, and the CS+ was 
no longer followed by a shock. For a graphical overview of the entire experimental procedure, see figure 1.

A)

\begin{tabular}{|c|c|c|c|c|}
\hline$\sim 8$ Minutes & $\sim 5$ Minutes & 10 Minutes & 15 Minutes & $\sim 4$ Minutes \\
\hline $\begin{array}{l}\text { Baseline HRV } \\
\text { and Habituation }\end{array}$ & $\begin{array}{l}\text { Acquisition } \\
12 \text { CS+ } 12 \text { CS- }\end{array}$ & $\begin{array}{l}\text { Questionnaires } \\
\text { STAI-S, STAI-T, PSWQ }\end{array}$ & 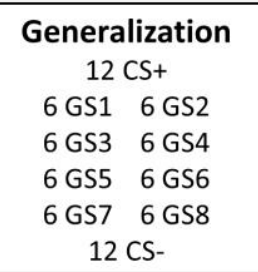 & $\begin{array}{l}\text { Extinction } \\
10 \mathrm{CS}+10 \mathrm{CS}-\end{array}$ \\
\hline & & & ental Mani & tion \\
\hline
\end{tabular}

B)

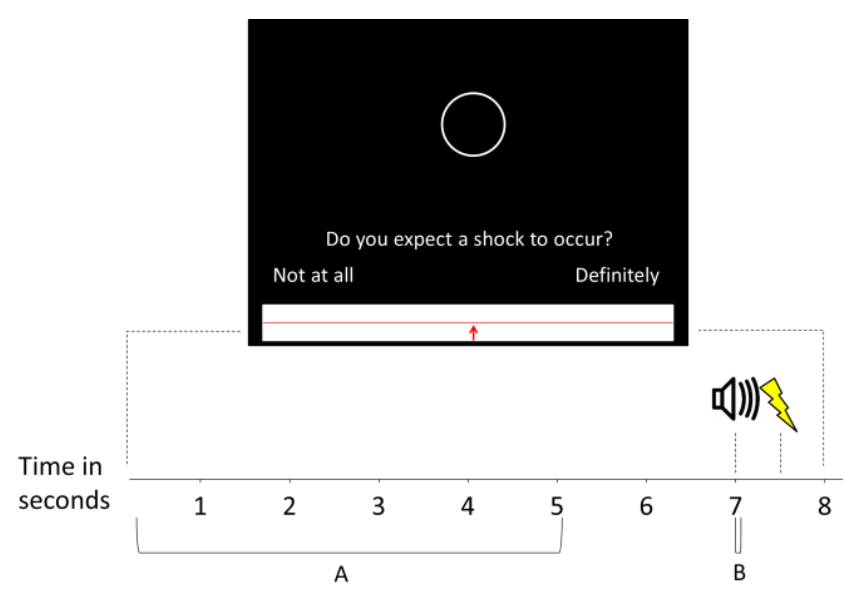

Figure 1. Experimental overview. A) The overall experiment lasted approximately 42 minutes. Participants received active $\mathrm{VVNS}$ or sham stimulation during the phases indicated by the grey frame. B) Trials lasted 8 seconds, followed by an intertrial interval lasting between 3.6 and 4.8 seconds. Participants were asked to rate to what extent they expected to receive a shock based on the stimulus that was being presented within the first 5 seconds (response window A). Startle probes presented after 7 seconds, and fear potentiated startle responses were recorded within $21-150 \mathrm{~ms}$ after startle probe onset (response window B). The US was presented for $20 \mathrm{~ms}, 7.5$ seconds after CS onset.

\section{$\underline{\text { Stimuli and Materials }}$}

\subsubsection{Conditioned and Unconditioned Stimuli}

The fear conditioning procedure was based on the methods used by Lissek and colleagues $[13,33]$. Specifically, 10 rings of gradually increasing size presented on a computer monitor 
were used as conditioned stimuli and generalization stimuli. The largest and smallest rings were assigned as CS+ and CS- trials, counterbalanced across participants. The eight intermediately sized rings served as generalization stimuli.

All conditioned and generalization stimuli were presented for 8 seconds, followed by an inter trial interval (ITI) varying in duration between 3.6 and $4.8 \mathrm{~s}$. The order of CS and GS presentation was quasi-randomized, allowing no more than 3 trials of the same type to appear in a row.

The unconditioned stimulus (US) was a 100-ms electrocutaneous stimulus delivered to the forearm of the non-dominant hand. The shock was delivered using a Grass S48 stimulator. The US was individually calibrated to an intensity that was 'highly uncomfortable, but not painful'. The US occurred 7.5s after CS+ onset.

Startle probes were presented during every CS and GS, 7s after stimulus onset. Additionally, startle probes were presented halfway through the ITI. Every startle probe consisted of a $40 \mathrm{~ms}$ loud broadband noise $(104 \mathrm{~dB})$ with an instantaneous rise and fall time.

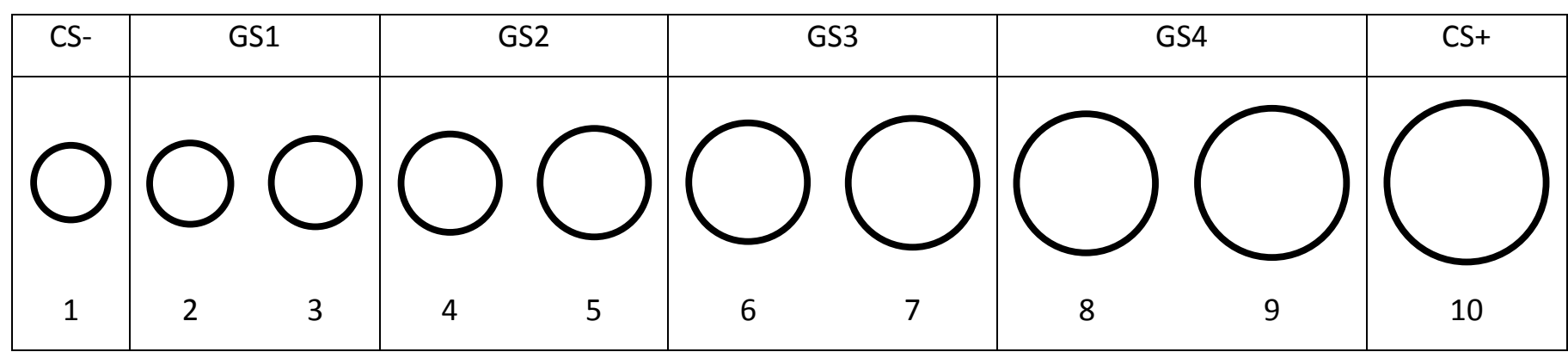

Figure 2. The smallest and largest circle served as CS- and CS+, while 8 circles of intermediate sizes were used as generalization stimuli. CS allocation was counterbalanced so that the smallest circle was the CS- for half of the participants and the CS+ for the other participants. GS categories were created by collapsing the eight intermediate ring sizes into four classes of generalization stimuli (GS1-GS4, GS4 being the category closest to the CS+). The diameter for the smallest ring was $2.1 \mathrm{~cm}$, and diameter size increased with $3 \mathrm{~mm}$ (roughly $15 \%$ increase compared to the smallest ring size, cf. [33]) for every subsequent ring up to $4.8 \mathrm{~cm}$ for the largest ring.

\subsection{2. tVNS and Sham Stimulation}

The tVNS instrument consisted of two titan electrodes mounted on a gel frame, connected to a wired neurostimulation device ( $\mathrm{CM02}$, Cerbomed, Erlangen, Germany). The VVNS $^{\circledR}$ 
device was programmed to a stimulus intensity of $0.5 \mathrm{~mA}$ with a stimulation frequency of 25 $\mathrm{Hz}$ and a stimulation wavelength of $250 \mu \mathrm{s}$. Stimulation was active for 30 seconds, followed by a break of 30 seconds. Participants started receiving stimulation after the acquisition phase, and it was continued for the remainder of the experiment.

Depending on their experimental allocation, participants received stimulation of either the cymba concha of the outer ear or the earlobe. The concha of the outer ear is $100 \%$ innervated by the vagus nerve [34]. Participants in the sham condition received stimulation on the earlobe, which is not innervated by the vagus nerve.

\section{3. $\quad$ Measurements}

\subsection{1. $E C G$}

Heart rate (HR) and heart rate variability (HRV) were derived from the raw ECG signal, which was measured continuously using a three-lead set-up of the Biopac system. The raw ECG signal was measured at $1000 \mathrm{~Hz}$ and subsequently filtered using $2 \mathrm{~Hz}$ low-pass and $50 \mathrm{~Hz}$ highpass software filters. The signal was subsequently visually inspected and artifacts were manually corrected. Interbeat intervals were extracted from the filtered signal, from which $\mathrm{HR}$ and the root mean square of the successive differences (RMSSD) between heart rates were calculated.

A five-minute baseline recording of every participant's RMSSD level was used to assess participants' vagally-mediated HRV and to check for possible differences in baseline vagal tone.

\subsubsection{Electromyography}

We measured the potentiation of the eyeblink startle reflex to an acoustic startle probe by using electromyography (EMG) of the left orbicularis oculi muscle. To measure the eyeblink reflex, we used two $4 \mathrm{~mm} \mathrm{Ag-AgCl} \mathrm{Biopac} \mathrm{electrodes,} \mathrm{one} \mathrm{placed} \mathrm{below} \mathrm{the} \mathrm{lower} \mathrm{left} \mathrm{eyelid}$ in line with the pupil in forward gaze, and the second one placed approximately $1 \mathrm{~cm}$ lateral to the first (in accordance with the guidelines specified by [35]). EMG was measured using a 
Biopac system, and filtered by $500 \mathrm{~Hz}$ low-pass and $10 \mathrm{~Hz}$ high-pass hardware filters. The EMG signal was grounded by the ECG electrodes.

EMG responses were calculated by subtracting the mean EMG signal in the $20 \mathrm{~ms}$ period directly following the startle probe presentation from the maximum EMG amplitude within the response window [35]. The response window was defined as the period between 21-150 ms following startle probe onset.

\subsubsection{US Expectancy Ratings}

Participants were asked to rate the extent to which they expected a shock to occur during every CS presentation using a visual analogue scale that ranged from 0 ('not at all') to 100 ('certainly'). Participants were instructed to give these ratings quickly since the rating scale would disappear from the screen 5 seconds after CS onset. The scale was presented at the bottom of the screen so as not to draw too much attention away from the stimuli. At the beginning of every new CS presentation, the slide would reappear and the cursor would return to the 'uncertain' middle position (cf. [36]).

\subsubsection{Questionnaires}

The State Trait Anxiety Inventory (STAI) is a questionnaire containing 2 scales with 20 questions each, measuring both state and trait anxiety $[37,38]$. The STAI has shown high internal consistency and validity [38,39]. The range of both scales of the STAI is $20-80$.

The Penn State Worry Questionnaire (PSWQ) is a 16-item self-report questionnaire that assesses the generality and uncontrollability of worry [40]. The PSWQ has demonstrated high reliability, temporal stability and validity in the assessment of trait-worry $[40,41]$. The range of the PSWQ is $16-80$.

\subsection{Statistical Analyses}


Between-group differences on all baseline questionnaires and baseline HRV data were analyzed using independent samples $t$-tests.

Multilevel mixed model analyses were used to assess whether the conditioning procedure resulted in successful fear learning in our participants in terms of both selfreports and physiological outcomes. All multilevel mixed models were created using maximum likelihood modeling. We allowed intercepts to vary randomly across participants. Random slopes were fitted to the models but did not improve model fit so were removed from all final models. Additionally, we modeled the nestedness of every trial within Stimulus within individual participants by specifying a heterogeneous $A R(1)$ autoregressive covariance structure of these repeated measurements.

For the analyses of the acquisition and extinction phases, the independent variable Trial, signifying trial number within each session, was group mean centered around the first trial of every phase. The independent variable Stimulus was dummy-coded, using CS- trials as the reference category for US expectancy ratings and using CS+ trials as the reference category for EMG, to allow comparisons of CS+ trials with both CS- trials and ITI.

To account for possible non-linear learning rates, we fitted linear and log transformed (In) time curves to all models, and removed either of these variables if this resulted in better model fit according to BIC estimates.

For the analyses of the generalization phase, GS categories were created by collapsing the eight intermediate ring sizes into four classes of generalization stimuli (see figure 2, cf. [13]). Trial number was based on the collapsed GS type presentation.

Generalization curve analyses were conducted according to the analysis recommendations detailed by VanBrabant and colleagues [42], analyzing the generalization curve as continuous scale ranging from non-threatening (CS-, 0$)$ to most threatening $(C S+, 5)$, with all intermittent GS types scoring in between (GS1, 1; GS2, 2; GS3, 3; GS4, 4).

All analyses concerning the effects of tVNS on extinction and generalization learning are reported as one-tailed tests to increase our power to detect an effect in the direction we expect. Analyses are conducted using the nlme and ImerTest packages in R. 


\section{Results}

\section{1. $\quad$ Demographics}

In total, 61 students participated in this study. Three participants were excluded from all analyses because of unchanging US expectancy ratings throughout the entire experiment. Thus, the final dataset consisted of 29 participants in the tVNS condition ( 21 female, 8 male, $M_{\text {age }}=22.1, S D_{\text {age }}=2.80$ ) and 29 participants in the sham condition (27 female, 2 male, $M_{\text {age }}$ $\left.=21.5, S D_{\text {age }}=1.99\right)$.

As displayed in table 1, there were no between-group differences on state or trait anxiety as indexed by the STAI or trait worry as indexed by the PSWQ. Mean scores on these questionnaires were similar to the mean scores of our previous study on the effects of tVNS on fear extinction [21]. Additionally, there were no significant baseline differences in heart rate or heart rate variability, as indexed by the natural log of RMSSD.

Table 1. Descriptive statistics. Mean scores on baseline variables with standard deviations presented between brackets.

\begin{tabular}{llll} 
& tVNS $(n=29)$ & Sham $(n=29)$ & $p$ \\
\cline { 2 - 4 } STAI-S & $43.8(9.9)$ & $45.8(10.1)$ & .44 \\
STAI-T & $41.0(9.0)$ & $38.7(8.6)$ & .32 \\
PSWQ & $46.9(9.0)$ & $46.0(8.8)$ & .70 \\
HR & $76.8(11.8)$ & & .28 \\
Ln RMSSD & $3.73(.55)$ & $3.51(.36)$ & .09 \\
\hline
\end{tabular}

Note: STAI = State-Trait Anxiety Inventory, $P S W Q=$ Penn State Worry Questionnaire, HR = Heart Rate, RMSSD = Root Mean Square of Successive Differences between heart rates. 


\subsection{Acquisition phase}

\subsubsection{US Expectancy}

US expectancy ratings of participants in both conditions reflected clear differential fear learning, as reflected by the LogTrial*Stimulus interaction, $b=28.78(S E=3.05), t(1328)=$ 9.44, $p<.001$. A significant effect of Stimulus indicates that this differential learning already started after the first trial, $b=15.77(5.92), t(1328)=2.67, p=.01$. US expectancy ratings for CS- trials diminished over the course of the Acquisition phase, as reflected by the main effect of LogTrial, $b=-12.96(2.16), t(1328)=-6.01, p<.001$.

There were no differences between the tVNS and Sham condition in US expectancy ratings during the Acquisition phase (all $p>.05$, see table 2 ).

\subsection{2. $E M G$}

Participants' fear potentiated startle responses reflected clear fear responses to the CS+ compared to the ITI, $b=-8.91(1.47), t(2034)=-6.08, p<.001$. However, participants only displayed a small, non-significant differential fear response to the CS+ compared to the CS-, $b=-1.74(1.47), t(2034)=-1.19, p=.24$. Regardless of Stimulus, participants showed a significant habituation of startle responses over the course of the Acquisition phase, as reflected by the main effect of Trial, $b=-.76(0.16), t(2034)=-4.63, p<.001$.

There were no differences between the tVNS and Sham condition in EMG responses during the Acquisition phase (all $p>.05$, see table 3 ).

\subsection{Generalization phase}

\subsubsection{US Expectancy}

Participants in both conditions showed a significant increase in US expectancy ratings for stimuli as a function of perceptual similarity to the CS+, as reflected by the effect of Stimulus, $b=10.79(0.84), t(58)=12.82, p<.001$ (see figure 3 ). There was no main effect of Condition on US expectancy ratings in this phase, $b=-2.87(3.77), t(58)=-.76, p=.45$, nor 
was there an effect of tVNS on the generalization gradient, reflected by the Condition*Stimulus Type interaction, $b=1.63$ (1.19), $t(58)=1.37, p=.18$.

\subsection{2. $E M G$}

Similarly to the US expectancy ratings, participants in both conditions showed a significant increase in EMG for stimuli as a function of perceptual similarity to the CS+, as reflected by the main effect of Stimulus, $b=1.26(0.16), t(61.46)=8.09, p<.001$. There was no main effect of Condition on EMG, $b=0.32(.56), t(61.53)=0.57, p=.57$, nor was there an effect of tVNS on the generalization gradient, reflected by the Condition*Stimulus interaction, $b=$ $0.11(0.22), t(61.33)=-0.51, p=.61$.
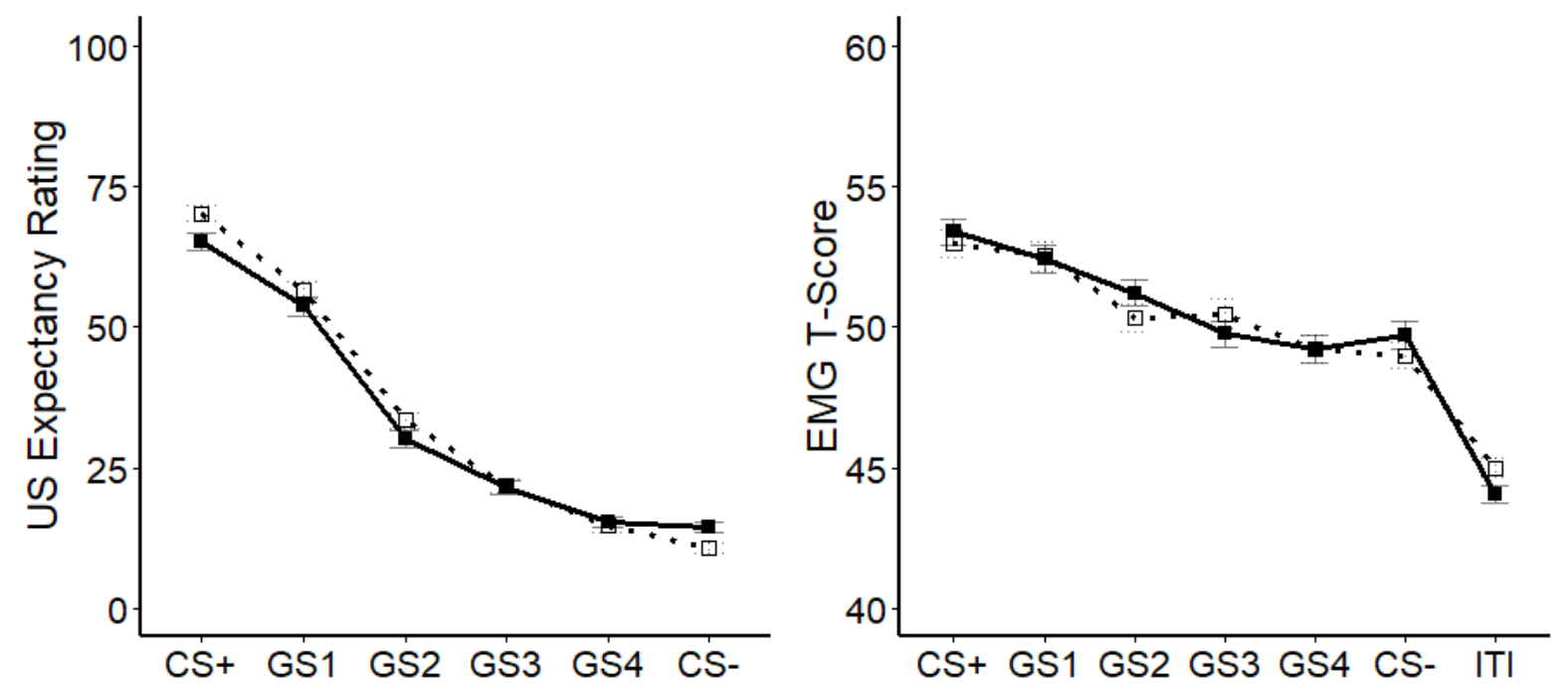

$-\mathrm{tVNS} \cdot \mathrm{S}$ Sham

Figure 3. A) US expectancy ratings for CS and GS stimuli during the generalization phase. B) Startle responses during CS and GS stimuli during generalization. Solid lines represent the tVNS condition, dotted lines denote the Sham condition. No significant between-group differences in fear generalization on either US expectancy ratings or EMG.

Error bars indicate \pm 1 standard error confidence intervals.

\subsection{Extinction phase}

\subsubsection{US Expectancy}

Over the course of the extinction phase, participants reported significantly higher US expectancy ratings for CS+ trials compared to CS- trials, $b=-68.22(4.76), t(1096)=14.32, p<$ 
.001. US expectancy ratings for CS- trials did not significantly change over the course of the extinction phase, as indicated by the main effect of Trial $(b=-.25(0.52), \mathrm{t}(1096)=-.48, p=$ .63). Similarly, the differential decline in US expectancy ratings for CS+ trials is only small and does not result in extinction of fear by the end of the extinction phase (see figure 4). This is also reflected in the non-significant Trial*Stimulus interaction, $b=-1.32(0.73), t(1096)=-$ $1.81, p=.07$.

As can be seen in figure 4, participants in the tVNS condition reported lower US expectancy ratings for $\mathrm{CS}+$ trials during the extinction phase, as reflected in the main effect of Condition, $b=-10.06(5.39), t(56)=-1.86, p=.03$. There was no significant difference in extinction learning curves between conditions, as reflected by the Trial*Stimulus*Condition interaction, $b=-.39(1.03), t(1096)=-.37, p=.71$.

\subsection{2. $E M G$}

Over the course of the extinction phase, participants displayed stronger EMG responses to the CS+ compared to the CS-, $b=-6.84$ (1.19), $t(1748)=-5.75, p<.001$, and compared to the ITI, $b=-10.34$ (1.19), $t(1748)=-8.70, p<.001$. Subsequently, participants showed a clear decline in EMG responses to the CS+, $b=-.60(0.16), t(1748)=-3.88, p<.001$. Importantly, this decline in EMG responses reflected a differential decline compared to CS- trials, $b=.36$ (0.22), $t(1748)=1.65, p=.10$, as well as ITI, $b=0.41(0.22), t(1748)=1.88, p=.06$.

There were no significant between-group differences on fear extinction as indexed by fear potentiated startle responses (all $p>.05$ ).

\section{Exploratory Analysis}

We conducted an additional exploratory analysis to test the effects of tVNS on RMSSD throughout the generalization and extinction phases. Specifically, we calculated the RMSSD of heart beats occurring within the first 7 seconds after every CS or GS onset. RMSSD scores were subsequently log transformed to ensure normal distribution of errors in the mixed model analysis. Visual inspection of the data showed no systematic differences in RMSSD between CStypes. Therefore, CStype was left out of the analysis. The final analysis consisted 
only of the main effects of Condition and Trial, and a Condition*Trial interaction. In this analysis, Trial is a continuous variable varying from 0 (first trial in the generalization phase) to 91 (last trial in the extinction phase).

There was a significant decrease in RMSSD over time, as indicated by the main effect of Trial, $b=-0.001(0.001), t(1549)=-2.20, p=.03$. There was no main effect of Condition on RMSSD, $b=0.15(0.19), t(65)=0.81, p=.42$, nor was there a significant Condition*Trial interaction, $b=-0.001(0.001), t(1549)=1.26, p=.21$.

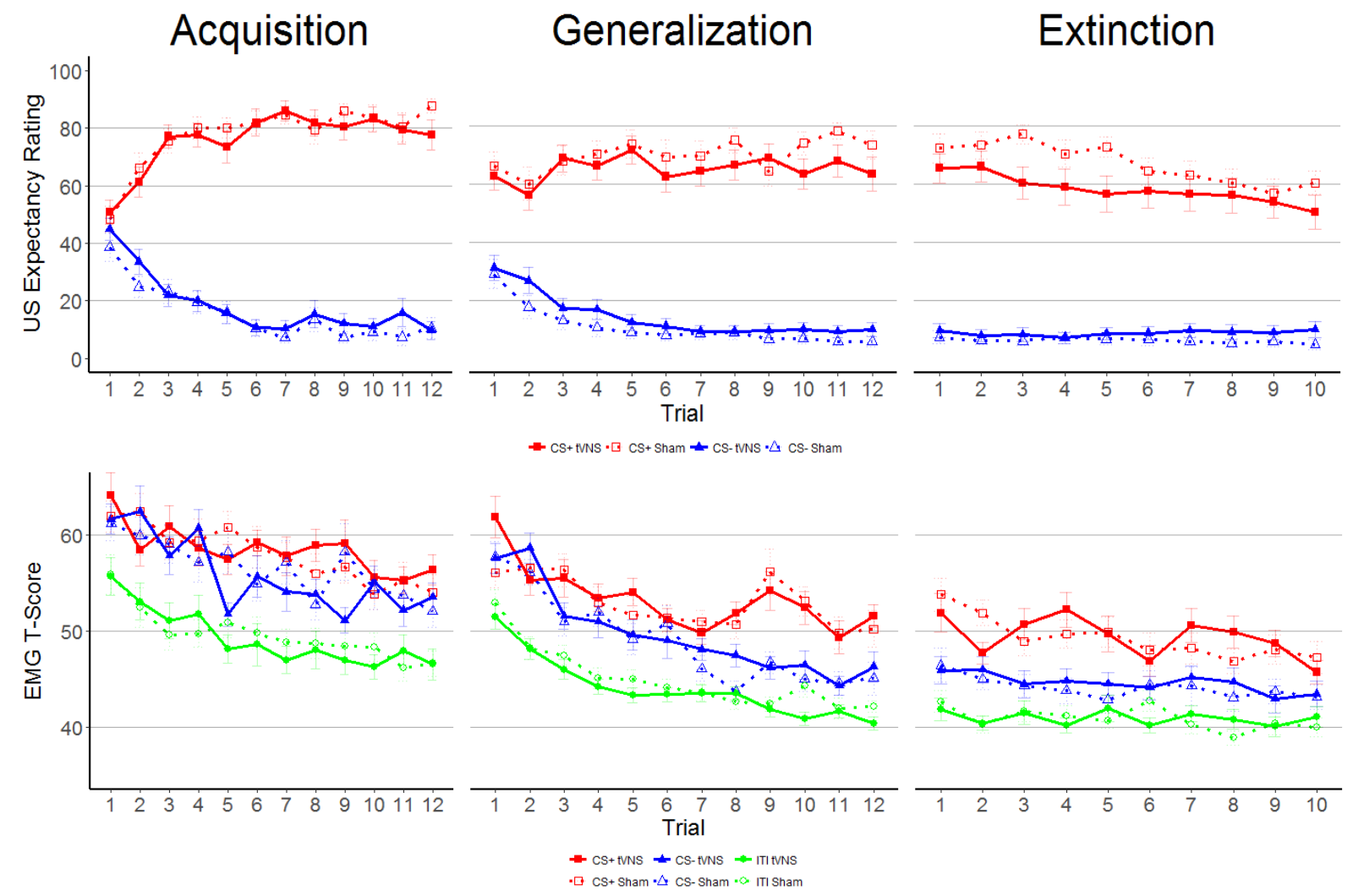

Figure 4. US expectancy ratings (top) and fear potentiated startle responses (bottom) during the fear acquisition (left), generalization (middle), and extinction (right). Only the CS+ (red), CS- (blue) and ITI (green) are represented in this figure. Solid lines represent the tVNS condition, dotted lines denote the Sham condition. Participants in the tVNS condition report significantly lower US expectancy ratings during the extinction phase compared to participants in the sham condition.

Generalization stimuli, presented only during the generalization phase, were omitted from these graphs to increase readability.

Error bars indicate \pm 1 standard error confidence intervals. 
Table 2. Regression weights and standard errors for mixed model analyses predicting US expectancy ratings in Acquisition, Generalization, and Extinction phases.

\begin{tabular}{llll}
\hline Predictor & Acquisition $^{\mathrm{a}}$ & Generalization & Extinction \\
\hline Intercept & $36.53(4.18)^{* * *}$ & $6.43(2.67)^{*}$ & $6.96(3.81)$ \\
Stimulus & $15.77(5.92)^{* * *}$ & $10.79(0.84)^{* * *}$ & $68.22(4.76)^{* * *}$ \\
Trial & $-12.96(2.16)^{* * *}$ & & $-0.25(0.52)$ \\
Trial*Stimulus & $28.78(3.05)^{* * *}$ & & $-1.32(0.72)$ \\
& & & \\
Condition & $4.98(5.92)$ & $-2.87(3.77)$ & $1.96(5.39)$ \\
Condition*Stimulus & $-1.14(8.37)$ & $1.63(1.19)$ & $-12.02(6.74)^{*}$ \\
Condition*Trial & $-1.41(3.05)$ & & $0.33(0.73)$ \\
Condition*Trial*Stimulus & $-2.41(4.31)$ & & $-0.39(1.03)$ \\
\hline
\end{tabular}

Note. Reference category for Stimulus is the CS- trial type. All analyses on the effects of tVNS were conducted using one-sided hypothesis tests. ${ }^{*} p<.05,{ }^{* *} p<.01,{ }^{* * *} p<.001$.

${ }^{a}$ Trial variable was log transformed to increase model fit for Acquisition data.

Table 3. Regression weights and standard errors for mixed model analyses predicting EMG in Acquisition, Generalization, and Extinction phases.

\begin{tabular}{|c|c|c|c|}
\hline Predictor & Acquisition & Generalization & Extinction \\
\hline Intercept & $61.91(1.37)^{* * *}$ & $46.17(.39)^{* * *}$ & $52.08(0.92)^{* * *}$ \\
\hline Stimulus & & $1.26(.16)^{* * *}$ & \\
\hline Stimuluscs- & $-1.62(1.50)$ & & $-6.87(1.21)^{* * *}$ \\
\hline Stimulus $_{\mid \mathrm{TI}}$ & $-8.84(1.50)^{* * *}$ & & $-10.42(1.21)^{* * *}$ \\
\hline Trial & $-0.71(0.17)^{* * *}$ & & $-0.59(0.16) * * *$ \\
\hline Trial*Stimulus cs- & $-0.01(0.22)$ & & $0.35(0.22)$ \\
\hline Trial*Stimulus & $0.07(0.22)$ & & $0.27(0.22)$ \\
\hline Condition & $-0.56(1.92)$ & $0.32(0.56)$ & $-1.54(1.29)$ \\
\hline Condition*Stimulus & & $-0.11(0.22)$ & \\
\hline Condition*Stimulus CS- & $0.50(2.11)$ & & $2.02(1.70)$ \\
\hline Condition*Stimulus $_{\mid \mathrm{TI}}$ & $0.39(2.11)$ & & $0.91(1.70)$ \\
\hline Condition*Trial & $0.20(0.23)$ & & $0.28(0.22)$ \\
\hline Condition*Trial*Stimulus cs- & $-0.23(0.31)$ & & $-0.31(0.31)$ \\
\hline Condition*Trial*Stimulus & $-0.21(0.31)$ & & $-0.12(0.31)$ \\
\hline
\end{tabular}

Note. Reference category for Stimulus is the CS+ trial type. All analyses on the effects of tVNS were conducted using one-sided hypothesis tests. ${ }^{*} p<.05,{ }^{* *} p<.01,{ }^{* * *} p<.001$. 


\section{Discussion}

The current study aimed to assess whether tVNS affects the generalization of fear in fear conditioned healthy students. The generalization gradient of physiological and declarative indices of fear did not significantly differ between the tVNS and sham conditions. Participants in the tVNS condition reported significantly lower US expectancy ratings for the CS+ stimuli over the course of the extinction phase. However, they did not differ from participants in the sham condition on physiological indices of fear during this phase. These latter results are in line with previous studies that have shown that stimulation of the vagus nerve may facilitate the extinction of declarative, but not physiological expressions of fear.

In the current study, we found no support for the hypothesis that tVNS reduces fear generalization. Given the evidence on the effects of VNS on hippocampal cell proliferation and differentiation from animal literature, it seemed likely that stimulating the vagus nerve in humans could have similar effects. The null results found in this study reflect the difficulty of translating animal studies to humans, and may also reflect differences between invasive and transcutaneous VNS. It should be noted, however, that the null results found in this study may also be due to the relatively short duration of tVNS in this study: not much is known about the temporal latency of the effects of VNS on progenitor proliferation or cell differentiation. In rats, acute effects on the increase in cell proliferation and dendritic complexity have only been tested after 3 hours of VNS. It is unclear whether these effects would also occur on a shorter timescale, such as the 30-minute timescale used in this experiment. It would be interesting to test whether possible effects of tVNS on the generalization of fear would appear after stimulating during multiple sessions. Currently, it remains unclear whether and how tVNS can be implemented in clinical practice for exposure therapy.

Participants in both conditions showed only limited extinction of fear during the extinction phase. The lack of complete extinction found in this study can be explained by the sudden change in CS-US contingency that occurred from the generalization phase to the extinction phase. During the generalization phase, the CS+ was followed by an electric shock in $50 \%$ of the trials, whereas the CS-US contingency dropped to $0 \%$ during the extinction phase. Since the generalization phase seamlessly transitioned into the extinction phase, the 
change in contingency from partial- to non-reinforcement was probably noticed by participants more slowly than when these phases would have been separated. Indeed, given the critical importance of expectancy violations for new learning to take place [43], lower CSUS contingencies during the generalization phase may have hampered expectancy violations and subsequent belief updating about the CS-US contingencies during the subsequent extinction phase [44]. To illustrate, after two CS-noUS trials, participants would be strongly inclined to update prior beliefs on CS-US contingencies if their prior belief was that the CS+ was followed by a US $100 \%$ of the time, whereas two non-reinforced trials would still fall in their realm of expectations when they held the prior belief that the CS+ was followed by a US 50\% of the time. Finally, it is important to note that the extinction phase contained only ten trials. If more trials had been included, it seems likely that participants in both conditions would have eventually shown complete extinction of fear.

Although on average participants in neither condition showed complete extinction of fear in this study, participants in the tVNS condition reported consistently lower US expectancy ratings for the CS+ over the course of the extinction phase. These results are in line with the general hypothesis that vagal nerve stimulation promotes the extinction of fear [45], although the accelerated declarative extinction curve that was found in earlier studies $[20,21]$ was not replicated. Differences between the current results and those from earlier studies may reflect differences between the underlying study designs. Specifically, as discussed in the previous paragraph, the generalization phase that preceded the extinction phase included a reduction in the CS-US contingency and thus already allowed for at least partial inhibitory learning to take place. Figure 4 shows that at the end of the generalization phase, participants in the tVNS condition already reported lower US expectancy ratings than participants who received sham stimulation, although this difference was not statistically significant. Throughout the subsequent extinction phase, participants in the tVNS condition retained these decreased US expectancy ratings, although extinction was not completed. In sum, given that partial inhibitory learning may have already taken place during the generalization phase, we believe that the Condition*CStype interaction found in the extinction phase is in line with the hypothesis that tVNS promotes the extinction of fear, and is also likely to reflect the more stringent hypothesis that tVNS accelerates the extinction of fear. As a cautionary note, however, we would like to point out that the Condition*CStype 
interaction that was found in the current study would no longer be significant when twotailed tests would have been utilized, indicating that the robustness and replicability of these results need to be determined in future studies.

In contrast to the declarative extinction of fear, participants in the tVNS condition did not differ from sham stimulated participants in their physiological fear responses during the generalization or the extinction phases. This is in line with previous studies on the effects of tVNS on fear extinction in humans [20,21]. Additionally, administration of the $\beta$-adrenergic receptor blocker propranolol during fear extinction has been found to significantly reduce the extinction of fear on declarative indices of fear, but did not affect physiological indices including fear potentiated startle responses and SCR [46]. The similarities between the effects of propranolol and tVNS in how they affect fear extinction provide further indications for the hypothesized noradrenergic working mechanism of tVNS.

The discrepant results of tVNS on declarative and physiological indices of fear could provide further support for a two-factor account of fear [47-49], which suggests that the declarative and emotional aspects of fear are controlled by two separate memory systems that are driven by separate brain areas (i.e. the hippocampus and the amygdala, respectively) [47]. Alternatively, the effect of tVNS on US expectancy ratings could reflect a non-emotional improvement in associative learning that is accelerated through tVNS, corresponding to earlier studies showing that tVNS strengthens associative learning of names and faces in older individuals [50]. Future studies should focus on whether the results found in this and in previous fear conditioning studies indeed extend towards non-emotional associative memory performance.

Although the fear generalization paradigm used in the present study has been commonly used in previous studies, there are two concerns with this method that we think should be addressed. Firstly, given that the only physical property distinguishing the CS+ from the CS- is the size of the circle, and no a priori knowledge on GS-US contingencies was available, it can theoretically be questioned if and to what extent the generalization of fear in this particular experimental paradigm can actually be described as maladaptive especially since the CS+ is still followed by a US $50 \%$ of the time, meaning that threat is still 
imminent. Secondly, it is also not entirely clear whether participants displayed a fear response because they feared the GS as such, that is, despite perceiving it as different from the $\mathrm{CS}+$, or because they were simply unable to discriminate the GS from the CS+. In a recent study, Struyf and colleagues [51] found that misspecification of the novel GS as the CS+ strongly increased fear responses compared to when the GS was categorized as a novel stimulation, and that misspecification of the GS as a CS+ occurs in up to $50 \%$ of trials depending on the similarity between stimuli in terms of circle size. These possible shortcomings of the experimental paradigm may explain why tVNS did not affect fear generalization in this trial, as the GSs could have been perceptually miss-specified as a CS+ and therefore no pattern completion or separation took place. Future studies could consider alternative paradigms which utilize CSs and GSs which are clearly distinguishable by testing, for example, conceptual generalization (e.g. the generalization of one type of bird to different types of birds).

The current study utilized an immediate extinction paradigm, with generalization and extinction phases occurring on the same day as fear acquisition. Although not much is known on the differences between immediate and delayed extinction, some reports indicate that immediate extinction is associated with delayed extinction learning (Chang \& Maren 2009, Maren 2014). One could argue that utilizing this immediate extinction paradigm increases the translational gap between animal and human studies, since animal studies studying the effects of VNS on fear extinction have exclusively used delayed learning paradigms. However, previous studies on the effects of tVNS on fear extinction in humans have utilized both immediate $[20,21]$ and delayed extinction protocols [22,23], and have found inconsistent results for both kinds of protocols. This may be due to other differences in the experimental protocol of these studies (e.g. cue vs. context conditioning, neutral vs. prepared learning). Clearly, there is a need for more consistency and more direct replications in this field of neurostimulation in extinction research.

Startle probes presented during CS or GS presentation were presented 7 seconds after stimulus onset. This fixed timing may have increased the predictability of the startle probe, and thereby decreased startle responses. This diminished startle responding may also partly explain why fear potentiated startle responses to CS+ stimuli were not significantly different from CS- stimuli during the acquisition phase. Indeed, preliminary evidence 
suggests that attending a startle probe diminishes the emotional modulation of the startle response, although this has only been studied when the startle probe required an active response [52]. The importance of startle probe onset predictability has not yet been systematically studied [30]. Nonetheless, future studies should consider presenting startle probes at random intervals relative to CS onset to circumvent the risk of attenuated startle responses to due stimulus predictability.

In the current study, we used the preprogrammed settings of the Nemos tVNS device of 30 seconds on, 30 seconds off stimulation at $25 \mathrm{~Hz}, 250 \mu$ s wavelength duration, with a stimulation intensity set to $0.5 \mathrm{~mA}$. One could argue that ensuring that stimulation occurs simultaneously with stimulus presentation is an important step towards optimizing treatment efficacy, given that (t)VNS is hypothesized to affect associative learning through phasic activation of the LC-NE network. Indeed, most studies that tested the effects of VNS on neuronal plasticity - as a way of treating tinnitus complaints or enhancing the rehabilitation for stroke patients - ensured that stimulation coincided with stimulus presentation (e.g. [53-57]). However, to our knowledge, there are no studies that have tested whether synchronization of stimulation and stimulus presentation is a determinant of the efficacy of $(t)$ VNS. In our earlier studies, we have found significant effects of tVNS on declarative fear extinction using stimulation paradigm that fixed the stimulation timing based on CS presentation [20] as well as when stimulation timing was purely based on a 30s on, 30s off schedule [21]. However, we have never tested both settings in one study before. Clearly, more research on the optimal stimulation parameters is clearly necessary, both in terms of stimulation intensity and frequency, but also in terms of timing of stimulation presentation.

Finally, we conducted an exploratory analysis to test the efferent cardiac effects of tVNS on RMSSD throughout the generalization and extinction phases. The potential for tVNS to elicit cardiac efferent effects is a contested issue, possibly fueled by the existing literature on HRV as a marker of cardiac vagal tone. In this study, tVNS did not affect participants' RMSSD compared to sham stimulation. Although there are some studies that have reported effects of tVNS on cardiac activity [58-60], the effects that have been reported are quite inconsistent. Additionally, studies that have found cardiac efferent effects of tVNS have used stimulation parameters that are quite different from the ones used in this study: firstly, they 
stimulated either the right ear or both ears, whereas we stimulated the left ear. Anatomical studies have pointed out that the right branch of the vagus nerve innervates the sinoatrial node of the heart more strongly than the left, which is also the reason why invasive VNS is most often applied to the left branch of the vagus nerve [61]. Secondly, studies that elicited cardiac efferent effects used stimulation intensities that were up to 10 times higher than the ones used in this study. This is in line with parametric studies performed in dogs, which showed that the stimulation intensities used in this study are sufficient to activate afferent A-fibers, but will not exceed the stimulation thresholds for activating cardiac efferent Bfibers (threshold of $0.4 \mathrm{~mA}$ and $3.8 \mathrm{~mA}$, respectively) [62,63].

To summarize, our hypothesis that tVNS affects the fear generalization gradient was not supported in this study. The risk of perceptual misspecification of CS and GS stimuli warrant a conceptual replication of this study using different stimuli. In line with previous studies, we observed an effect of tVNS on the extinction of declarative fear and no effect on physiological fear extinction. These findings support the theory that tVNS affects associative learning performance. 


\section{Acknowledgments}

We would like to thank Silvia Bucciarelli for her help during data collection. This work was supported by research grants from the Netherlands Organization for Scientific Research (NWO) awarded to Bart Verkuil (Veni Grant 451-14-013).

Declarations of interest: none. 


\section{References}

[1] Duits P, Cath DC, Lissek S, Hox JJ, Hamm AO, Engelhard IM, et al. Updated metaanalysis of classical fear conditioning in the anxiety disorders. Depress Anxiety 2015;32:239-53. doi:10.1002/da.22353.

[2] Lissek S, Powers A, McClure E, Phelps E, Woldehawariat G, Grillon C, et al. Classical fear conditioning in the anxiety disorders: a meta-analysis. Behav Res Ther 2005;43:1391-424. doi:10.1016/j.brat.2004.10.007.

[3] Dymond S, Dunsmoor JE, Vervliet B, Roche B, Hermans D. Fear Generalization in Humans: Systematic Review and Implications for Anxiety Disorder Research. Behav Ther 2015;46:561-82. doi:10.1016/j.beth.2014.10.001.

[4] Yassa MA, Stark CEL. Pattern separation in the hippocampus. Trends Neurosci 2011;34:515-25. doi:10.1016/j.tins.2011.06.006.Pattern.

[5] Besnard A, Sahay A. Adult Hippocampal Neurogenesis, Fear Generalization, and Stress. Neuropsychopharmacology 2016;41:24-44. doi:10.1038/npp.2015.167.

[6] Neunuebel JP, Knierim JJ. CA3 Retrieves Coherent Representations from Degraded Input: Direct Evidence for CA3 Pattern Completion and Dentate Gyrus Pattern Separation. Neuron 2014;81:416-27. doi:10.1016/j.neuron.2013.11.017.CA3.

[7] Maren S, Aharonov G, Fanselow MS. Neurotoxic lesions of the dorsal hippocampus and Pavlovian fear conditioning in rats. Behav Brain Res 1997;88:261-74. doi:10.1016/S0166-4328(97)00088-0.

[8] Antoniadis EA, McDonald RJ. Amygdala, hippocampus and discriminative fear conditioning to context. Behav Brain Res 2000;108:1-19. doi:10.1016/S01664328(99)00121-7.

[9] Frankland PW, Cestari V, Filipkowski RK, McDonald RJ, Silva AJ. The dorsal hippocampus is essential for context discrimination but not for contextual conditioning. Behav Neurosci 1998;112:863-74.

[10] Gilbert PE, Kesner RP, Lee I. Dissociating hippocampal subregions: A double 
dissociation between dentate gyrus and CA1. Hippocampus 2001;11:626-36. doi:10.1002/hipo.1077.

[11] McHugh TJ, Jones MW, Quinn JJ, Balthasar N, Coppari R, Elmquist JK, et al. Dentate Gyrus NMDA Receptors Mediate Rapid Pattern Separation in the Hippocampal Network. Science (80- ) 2007;317:94-9. doi:10.1126/science.1140263.

[12] Bakker A, Kirwan CB, Miller M, Stark CEL. Pattern Separation in the Human Hippocampal CA3 and Dentate Gyrus. Science (80- ) 2010;319:1640-2. doi:10.1126/science.1152882.Pattern.

[13] Lissek S, Bradford DE, Alvarez RP, Burton P, Espensen-sturges T, Reynolds RC, et al. Neural substrates of classically conditioned fear-generalization in humans : a parametric fMRI study. Scan 2014;9:1134-42. doi:10.1093/scan/nst096.

[14] Ruffoli R, Giorgi FS, Pizzanelli C, Murri L, Paparelli A, Fornai F. The chemical neuroanatomy of vagus nerve stimulation. J Chem Neuroanat 2011;42:288-96. doi:10.1016/j.jchemneu.2010.12.002.

[15] Klarer M, Arnold M, Günther L, Winter C, Langhans W, Meyer U. Gut vagal afferents differentially modulate innate anxiety and learned fear. J Neurosci 2014;34:7067-76. doi:10.1523/JNEUROSCI.0252-14.2014.

[16] Peña DF, Childs JE, Willett S, Vital A, McIntyre CK, Kroener S. Vagus nerve stimulation enhances extinction of conditioned fear and modulates plasticity in the pathway from the ventromedial prefrontal cortex to the amygdala. Front Behav Neurosci 2014;8:327. doi:10.3389/fnbeh.2014.00327.

[17] Peña DF, Engineer ND, McIntyre CK. Rapid remission of conditioned fear expression with extinction training paired with vagus nerve stimulation. Biol Psychiatry 2013;73:1071-7. doi:10.1016/j.biopsych.2012.10.021.

[18] Noble LJ, Gonzalez IJ, Meruva VB, Callahan KA, Belfort BD, Ramanathan KR, et al. Effects of vagus nerve stimulation on extinction of conditioned fear and posttraumatic stress disorder symptoms in rats. Transl Psychiatry 2017;7:e1217. doi:10.1038/tp.2017.191. 
[19] Alvarez-Dieppa AAC, Griffin K, Cavalier S, Mcintyre CK. Vagus nerve stimulation enhances extinction of conditioned fear in rats and modulates Arc protein, CaMKII, and GluN2B-containing NMDA receptors in the basolateral amygdala. Neural Plast 2016:1-19.

[20] Burger AM, Verkuil B, Fenlon H, Thijs L, Cools L, Miller HC, et al. Mixed evidence for the potential of non-invasive transcutaneous vagal nerve stimulation to improve the extinction and retention of fear. Behav Res Ther 2017;97:64-74. doi:10.1016/j.brat.2017.07.005.

[21] Burger AM, Verkuil B, Van Diest I, Van der Does W, Thayer JF, Brosschot JF. The effects of transcutaneous vagus nerve stimulation on conditioned fear extinction in humans. Neurobiol Learn Mem 2016;132:49-56. doi:10.1016/j.nlm.2016.05.007.

[22] Burger AM, Van Diest I, van der Does W, Hysaj M, Thayer JF, Brosschot JF, et al. Transcutaneous vagus nerve stimulation and extinction of prepared fear: A conceptual non-replication. Sci Rep 2018;8:11471. doi:10.1038/s41598-018-29561-w.

[23] Genheimer H, Andreatta M, Asan E, Pauli P. Reinstatement of contextual conditioned anxiety in virtual reality and the effects of transcutaneous vagus nerve stimulation in humans. Sci Rep 2017;7:17886. doi:10.1038/s41598-017-18183-3.

[24] Segal SK, Stark SM, Kattan D, Stark CE, Yassa MA. Norepinephrine-mediated emotional arousal facilitates subsequent pattern separation. Neurobiol Learn Mem 2012;97:465-9. doi:10.1016/j.nlm.2012.03.010.

[25] Revesz D, Tjernstrom M, Ben-Menachem E, Thorlin T. Effects of vagus nerve stimulation on rat hippocampal progenitor proliferation. Exp Neurol 2008;214:25965. doi:10.1016/j.expneurol.2008.08.012.

[26] Biggio F, Gorini G, Utzeri C, Olla P, Marrosu F, Mocchetti I, et al. Chronic vagus nerve stimulation induces neuronal plasticity in the rat hippocampus. Int J Neuropsychopharmacol 2009;12:1209-21. doi:10.1017/S1461145709000200.

[27] Gebhardt N, Bär KJ, Boettger MK, Grecksch G, Keilhoff G, Reichart R, et al. Vagus nerve stimulation ameliorated deficits in one-way active avoidance learning and stimulated hippocampal neurogenesis in bulbectomized rats. Brain Stimul 2013;6:78-83. 
doi:10.1016/j.brs.2012.01.009.

[28] Davis M. Neural systems involved in fear and anxiety measured with fear-potentiated startle. Am Psychol 2006;61:741-56. doi:10.1037/0003-066X.61.8.741.

[29] Davis M, Falls WA, Campeau S, Kim M. Fear-potentiated startle: a neural and pharmacological analysis. [Review]. Behav Brain Res 1993;58:175-98.

[30] Lonsdorf TB, Menz MM, Andreatta M, Fullana MA, Golkar A, Haaker J, et al. Don't fear 'fear conditioning': Methodological considerations for the design and analysis of studies on human fear acquisition, extinction, and return of fear. Neurosci Biobehav Rev 2017;77:247-85. doi:10.1016/j.neubiorev.2017.02.026.

[31] Soeter M, Kindt M. Dissociating response systems: erasing fear from memory. Neurobiol Learn Mem 2010;94:30-41. doi:10.1016/j.nlm.2010.03.004.

[32] Frangos E, Ellrich J, Komisaruk BR. Non-invasive Access to the Vagus Nerve Central Projections via Electrical Stimulation of the External Ear: fMRI Evidence in Humans. Brain Stimul 2014;8:624-36. doi:10.1016/j.brs.2014.11.018.

[33] Lissek S, Biggs AL, Rabin SJ, Cornwell BR, Alvarez RP, Pine DS, et al. Generalization of conditioned fear-potentiated startle in humans: Experimental validation and clinical relevance. Behav Res Ther 2008;46:678-87. doi:10.1016/j.brat.2008.02.005.

[34] Peuker ET, Filler TJ. The nerve supply of the human auricle. Clin Anat 2002;15:35-7. doi:10.1002/ca.1089.

[35] Blumenthal TD, Cuthbert BN, Filion DL, Hackley S, Lipp O V., Van Boxtel A. Committee report: Guidelines for human startle eyeblink electromyographic studies. Psychophysiology 2005;42:1-15. doi:10.1111/j.1469-8986.2005.00271.x.

[36] Kindt $M$, Soeter $M$, Vervliet B. Beyond extinction: erasing human fear responses and preventing the return of fear. Nat Neurosci 2009;12:256-8. doi:10.1038/nn.2271.

[37] van der Ploeg HM. De Zelf-Beoordelings Vragenlijst (STAI-DY). De ontwikkeling en validatie van een Nederlandstalige vragenlijst voor het meten van angst. Tijdschr Psychiatr 1982;24:576-88.

[38] Spielberger CD, Gorsuch RL, Lushene RE. STAI Manual for the State-Trait Anxiety 
Inventory. Palo Alto: Consulting Psychologists Press; 1970.

[39] Barnes LLB, Harp D, Jung WS. Reliability Generalization of Scores on the Spielberger State-Trait Anxiety Inventory. Educ Psychol Meas 2002;62:603-18. doi:10.1177/0013164402062004005.

[40] Meyer TJ, Miller ML, Metzger RL, Borkovec TD. Development and validation of the Penn State Worry Questionnaire. Behav Res Ther 1990;28:487-95. doi:10.1016/00057967(90)90135-6.

[41] Verkuil B, Brosschot J, Thayer J. Capturing worry in daily life: Are trait questionnaires sufficient? Behav Res Ther 2007;45:1835-44. doi:10.1016/j.brat.2007.02.004.

[42] Vanbrabant K, Boddez Y, Verduyn P, Mestdagh M, Hermans D, Raes F. A new approach for modeling generalization gradients: a case for hierarchical models. Front Psychol 2015;6:652. doi:10.3389/fpsyg.2015.00652.

[43] Rescorla RA, Wagner AR. A theory of Pavlovian conditioning: Variations in the effectiveness of reinforcement and nonreinforcement. Class Cond II Curr Res Theory 1972;21:64-99. doi:10.1101/gr.110528.110.

[44] Grady AK, Bowen KH, Hyde AT, Totsch SK, Knight DC. Effect of continuous and partial reinforcement on the acquisition and extinction of human conditioned fear. Behav Neurosci 2016;130:36-43. doi:10.1037/bne0000121.

[45] McIntyre CK. Is there a role for vagus nerve stimulation in the treatment of posttraumatic stress disorder? Bioelectron Med 2018;1:95-9. doi:10.2217/bem-20180002.

[46] Bos MGN, Beckers T, Kindt M. The effects of noradrenergic blockade on extinction in humans. Biol Psychol 2012;89:598-605. doi:10.1016/j.biopsycho.2012.01.007.

[47] Phelps E. Human emotion and memory: Interactions of the amygdala and hippocampal complex. Curr Opin Neurobiol 2004;14:198-202. doi:10.1016/j.conb.2004.03.015.

[48] LeDoux JE. Coming to terms with fear. Proc Natl Acad Sci 2014;111:2871-8. doi:10.1073/pnas.1400335111. 
[49] Hamm AO, Vaitl D, Lang PJ. Fear conditioning, meaning, and belongingness: A selective association analysis. J Abnorm Psychol 1989;98:395-406. doi:10.1037/0021843X.98.4.395.

[50] Jacobs HIL, Riphagen JM, Razat CM, Wiese S, Sack AT. Transcutaneous vagus nerve stimulation boosts associative memory in older individuals. Neurobiol Aging 2015;36:1860-7. doi:10.1016/j.neurobiolaging.2015.02.023.

[51] Struyf D, Zaman J, Hermans D, Vervliet B. Gradients of fear: How perception influences fear generalization. Behav Res Ther 2017;93:116-22. doi:10.1016/j.brat.2017.04.001.

[52] Panayiotou G, van Oyen Witvliet C, Robinson JD, Vrana SR. Modulation of the Startle Reflex By Valence and Arousal. Biol Psychol 2012;87:226-33. doi:10.1016/j.biopsycho.2011.03.001.A.

[53] Engineer ND, Riley JR, Seale JD, Vrana W a, Shetake J a, Sudanagunta SP, et al. Reversing pathological neural activity using targeted plasticity. Nature 2011;470:1014. doi:10.1038/nature09656.

[54] Meyers EC, Solorzano BR, James J, Ganzer PD, Lai ES, Rennaker RL, et al. Vagus nerve stimulation enhances stable plasticity and generalization of stroke recovery. Stroke 2018;49:710-7. doi:10.1161/STROKEAHA.117.019202.

[55] Vanneste S, Martin J, Rennaker RL, Kilgard MP. Pairing sound with vagus nerve stimulation modulates cortical synchrony and phase coherence in tinnitus: An exploratory retrospective study. Sci Rep 2017;7:17345. doi:10.1038/s41598-01717750-y.

[56] Borland MS, Engineer CT, Vrana WA, Moreno NA, Engineer ND, Vanneste S, et al. The Interval Between VNS-Tone Pairings Determines the Extent of Cortical Map Plasticity. Neuroscience 2018;369:76-86. doi:10.1016/j.neuroscience.2017.11.004.

[57] Tyler R, Cacace A, Stocking C, Tarver B, Engineer N, Martin J, et al. Vagus Nerve Stimulation Paired with Tones for the Treatment of Tinnitus: A Prospective Randomized Double-blind Controlled Pilot Study in Humans. Sci Rep 2017;7:1-11. doi:10.1038/s41598-017-12178-w. 
[58] Clancy J a, Mary D a, Witte KK, Greenwood JP, Deuchars S a, Deuchars J. Non-invasive Vagus Nerve Stimulation in Healthy Humans Reduces Sympathetic Nerve Activity. Brain Stimul 2014. doi:10.1016/j.brs.2014.07.031.

[59] Badran BW, Mithoefer OJ, Summer CE, LaBate NT, Glusman CE, Badran AW, et al. Short trains of transcutaneous auricular vagus nerve stimulation (taVNS) have parameter-specific effects on heart rate. Brain Stimul 2018;11:699-708. doi:10.1016/j.brs.2018.04.004.

[60] Couck M De, Cserjesi R, Caers R, Zijlstra WP, Widjaja D, Wolf N, et al. Effects of short and prolonged transcutaneous vagus nerve stimulation on heart rate variability in healthy subjects. Auton Neurosci Basic Clin 2017;203:88-96. doi:10.1016/j.autneu.2016.11.003.

[61] Ardell JL, Randall WC. Selective vagal innervation of sinoatrial and atrioventricular nodes in canine heart. Am J Physiol 1986;251:H764-73.

[62] Yoo PB, Liu H, Hincapie JG, Ruble SB, Hamann JJ, Grill WM. Modulation of heart rate by temporally patterned vagus nerve stimulation in the anesthetized dog. Physiol Rep 2016;4:1-10. doi:10.14814/phy2.12689.

[63] Yuan H, Silberstein SD. Vagus Nerve and Vagus Nerve Stimulation, a Comprehensive Review: Part I. Headache 2016;56:71-8. doi:10.1111/head.12647. 